\title{
Tandem repeats are selfish elements which mark the level of hidden recombination in animal mitochondrial genomes
}

\author{
Aleksandr Smirnov \\ Center for Mitochondrial Functional \\ Genomics \\ Immanuel Kant Baltic Federal \\ University \\ Kaliningrad, Russia \\ aleksandr.smirnov2009@gmail.com \\ Konstantin Gunbin \\ Institute of Cytology and Genetics \\ SB \\ RAS, Novosibirsk, Russia \\ Immanuel Kant Baltic Federal \\ University \\ Kaliningrad, Russia \\ genkvg@gmail.com
}

\author{
Alina A. Mikhailova \\ Center for Mitochondrial Functional \\ Genomics, Immanuel Kant Baltic \\ Federal University \\ Kaliningrad, Russia \\ mihailovaalina777@yandex.ru \\ Konstantin Popadin \\ School of Life Sciences \\ Ecole Polytechnique Federale de \\ Lausanne, Lausanne, Switzerland \\ Center for Mitochondrial Functional \\ Genomics, Immanuel Kant Baltic \\ Federal University Kaliningrad, \\ Russia \\ konstantinpopadin@gmail.com
}

\author{
Valeria Lobanova \\ Center for Mitochondrial Functional \\ Genomics, Immanuel Kant Baltic \\ Federal University \\ Kaliningrad, Russia \\ aaren183@gmail.com
}

\begin{abstract}
Despite the fact that mitochondrial genome is streamlined, its size still varies significantly across all vertebrate taxa (from 15kb in Mammalia to $25 \mathrm{~kb}$ in Reptilia approximately).

To uncover the driving reason of this variation we analyzed structures of the whole mitochondrial genomes of 3793 Chordata species belonging to 5 taxa: Actinopterygii (1905 sp.), Amphibia (227 sp.), Reptilia (285 sp.), Mammalia (855 sp.), and Aves (521 sp.). In each of these taxa we observed strong positive correlation between the genome size and the length of non-coding region (D-loop) (all Spearman's rho $>=$ 0.76 , all $p$-values $<=2.2 \mathrm{e}-16$ ). We assumed that some repeated sequences may contribute in D-loop length variation and looked for five types or repeats: tandem (annotated by Tandem Repeats Finder), direct, symmetrical, inverted and complementary (annotated by our own scripts). Analyzing the impact of the number and the length of each repeat type on $D$ loop size variation we revealed that the tandem repeats is the main repeat types, shaping the size of the D-loop.
\end{abstract}

\section{Keywords - Tandem repeats, recombination}

\section{Introduction}

To reveal potential associations between the abundance of the tandem repeats and species-specific life-history traits we focused on Mammals, for majority of which there is a high quality ecological information such as generation length, body mass and metabolic rate. For 434 species we observed a negative correlation between tandem repeats abundance and generation length (rho $=-0.10, \mathrm{p}$-value $=$ 0.029 ) indicating that tandem repeats are more common in short-lived species (this correlation is robust if we introduce in our model GC content and PIC).

It is important to take into account not only presence/absence of tandem repeats and their total length but also properties of their motifs: length of the motif, nucleotide content of the motif, copy number of the motif (how many times it is repeated in mtDNA), the level of degradation (average similarity between each repeated copy of the motif), and so on. Using pairwise correlations and principal component analysis we observed several groups of tandem repeats, for example more GC rich motifs tend to be more perfect (less degraded) with more repeated copies. We hypothesized that some of these groups of repeats may be more important than others in the shaping of the correlation with ecological (generation length) and genetical (whole genome size, D-loop size) traits. Indeed we observed that long, GC-rich, low-degraded and many-times-repeated motifs correlate better with generation time.

There are three non-mutually exclusive potential explanations of the observed negative correlation between the tandem repeats abundance and generation time: (i) strong negative selection against tandem repeats in mtDNA of longlived species (within long-lived dormant oocytes)[2], (ii) selfish drive of tandem repeats and (iii) neutral propagation of tandem repeats in mtDNA of species with non-zero level of recombination.

\section{Methods}

(i) It is known that in human postmitotic tissues (neurons and skeletal muscles) somatic mtDNA deletions propagate with age leading to many age-related phenotypes. Somatic deletions are very deleterious and are almost never transmitted from mother to offspring but probably the same process is happening on the low scale during evolution. This process is expected to be more powerful in the oocyte, which lives long life - for example in elephants it is expected to be stronger than in mice ( 20 years within elephant oocyte versus 1 year in mouse oocyte). However, until now there is a lack of evidence, that short size of the genome is associated with clonal advantage.

(ii) Tandem repeats may provide an advantage to the host mtDNA (selfish drive)activating replication of a given molecule. Recently it has been shown that some tandem repeats may increase replication rate of the host mtDNA genome providing it a replication advantage [3]. Interestingly this process may lead to the accumulation of deleterious variants throughout the whole genome (and even to extinction of a line) since replication advantage overcomes some deleterious effects of the linked variants [3]. In this scenario, tandem repeats are active drivers of the increased replication rate of the host molecules. 
(iii) Tandem repeats (especially with long motifs) may propagate better in the recombinational environment and thus they might be a neutral marker of the hidden recombination. It is commonly accepted that mtDNA of chordata are mainly non-recombining, maternally inherited genome. However, numerous well-proofed exceptions are known from this rule. We hypothesize, that tandem repeats can be a marker of the low level of recombination, undetectable by many other methods.

Interestingly the number and the length of tandem repeats differ not only between species but also between taxa: on the average tandem repeats are more common in Amphibia and Reptilia while genomes of Mammalia and Aves are more poor in tandem repeats. It is known that exactly Amphibia have the highest frequency of biparental inheritance of mtDNA.

Altogether we conclude that tandem repeats are markers of hidden and rare recombination in mtDNA of vertebrate species Additionally we decided to estimate the probability of recombination in mammalian species using polymorphism data. For 224 mammalian species we were able to find 10 different CytB sequences, aligned them and analyzed a probability of recombination using LDr2 approach

(http://www.lifesci.sussex.ac.uk/CSE/test/ld.php). After analyzing polymorphic data of 224 species for 33 of them we found some evidence of recombination (species with nominal p-values less or equal 0.01). Next we tested if recombination is associated with tandem repeats. We observed that among species with more than one tandem repeat the fraction of species with recombination is $21 \%$ (17 out of 80 ) while among the species with one or less tandem repeats this fraction is only $11 \%$ (16 out of 128 species). The increased fraction of recombination among the tandemrepeat-rich species is marginally significant $(\mathrm{p}=0.049$, Fisher's odds ratio $=2.15)$. Interestingly, between class comparison showed that aves have the most frequent recombination ( $18 \%$ of species), actinopterygii - the second most frequent $(11 \%)$ while reptilia $(8 \%)$ and mammals $(7 \%)$ are the classes with the most rare recombination detected (we don't have enough amphibia species to validate it). In the future we plan to use several additional tests on recombination in order to validate the robustness of our findings.

\section{Results}

Altogether, using several lines of evidence (association of tandem repeats with mtDNA genome length, with speciesspecific generation length, with $\mathrm{Kn} / \mathrm{Ks}$ and with recombination events) we conclude that mtDNA tandem repeats indeed mark very rare and very

hidden recombination.

\section{Acknowledgment}

This work is supported by the 5 Top 100 Russian Academic Excellence Project at the Immanuel Kant Baltic Federal University, and by the Russian Foundation for Basic Research grant 18-29-13055.

\section{References}

[1] M. Pacifici, et al. (2013) Generation length for mammals, Nature Conservation, 5:89.

[2] Y. Kraytsberg, et al. (2006) Mitochondrial DNA deletions are abundant and cause functional impairment in aged human substantia nigra neurons, Nature genetics, 38.5:518;

[3] H. Ma, and P. H. O'Farrell (2016) Selfish drive can trump function when animal mitochondrial genomes compete, Nature genetics, 48.7:798,

[4] L. Polishchuk, et al. (2015) A genetic component of extinction risk in mammals, Oikos, 124.8:983-993;

[5] D. J. White, and N. J. Gemmell (2009) Can Indirect Tests Detect a Known Recombination Event in Human mtDNA?, Molecular Biology and Evolution, 26.7:1435 